\title{
Power estimation in Array Signal Processing: Case study
}

\author{
Youssef Khmou \\ Sultan Moulay Slimane University, Morocco. \\ khmou.y@gmail.com
}

\begin{abstract}
Characterizing radiating source by an array of sensors is an important process in many scientific and civil applications such optical interferometry, telecommunications, seismology and submarine acoustics. When the collected echoes by the array are a mixture of several sources, processing data needs to take into account the interactions between the individual components such as spatial coherence or correlation between primary sources, interferences from secondary sources as perturbing noise and power level of each wavefront. In the case of single emitter, the problem of source characterization becomes easy, because a simple treatment of second order statistics of received data is sufficient to identify the parameters of the source such as the angle of incidence, the shape of the waveform and its variance or power. In this paper, we propose an alternative approach of power estimation of single source using an array of isotropic sensors, the conditions used are those of the far field radiation making the approximation of planar wave front valid and the narrowband spectrum where the bandwidth of waveform is negligible against the carrier frequency. We propose the combination of the standard beam forming spectrum and the information that diagonal elements of spatial covariance matrix contains, the solution of Signal to Noise Ratio estimation is based on two dimensional linear system. Some numerical simulations are carried out to compare the performance of the proposed solution with the method based on eigenvalues computation.
\end{abstract}

Keywords: Signal to Noise Ratio (SNR), array of sensors, power estimation, narrowband far-field

\section{Introduction}

Wave propagation $[1,2]$ mechanism is an essential process to understand in order to characterize a waveform [3], with different metrics including the source of propagation, the frequency range, the polarization [4], the energy or power in the duration of observation, the source's envelope, the variation of the phase and so on. Waveform estimation is generally made using a collection of sensors that placed in different positions so as to estimate the variations of wave's characteristics in region of space, the process of estimation becomes difficult in the presence of multiple sources [5]. In this case, the interference [6] has an impact of the waveform characterization where it is mandatory to statistically separate the sources. Another perturbing factor is the additive noise generated by internal electronic circuits of the sensors or by external sources of propagation, to eliminate the noise, statistical relations between signal and noise permit to cancel the effect of perturbation using at least second order statistics of collected data.

Given the condition that the collected data is perturbed by an additive noise which is generally described by Gaussian distribution with zero mean, the power estimation of radiating sources is measured relatively to noise level, the metric of

Received (July 5, 2017), Review Result (October 23, 2017), Accepted (October 26, 2017) 
analysis is the signal to noise ratio (SNR) [7] which is logarithmic function that describes the state of collected data where processing the waveform characteristics is valid if the SNR is sufficiently high for better estimation. In the case of multiple sources that have the same power, the data is a superposition of elementary waveforms and the matrix measurement by sensors can be defined by unique SNR, in other scenarios where the present sources have different powers, the SNR varies. To simulate the experimental acquisition of data, performance of waveforms estimation needs to consider the different values of powers, because in the case of unique SNR, simulation becomes easy to evaluate the metrics with one vector of SNR. In this particular case of one waveform, a simple analysis of covariance matrix permits to estimate the SNR, by examining the eigenvalues of covariance matrix [1] of collected data, a precise estimation of source power is possible, this process is included in the methods of subspace based angular scans $[1,8]$.

In this paper we demonstrate another solution of power estimation [9] of one radiating source, the mechanism is based on the peak value of standard beam forming spectrum and the trace of spatial covariance matrix. In the second section, we present the data model of the array in the case of one radiating source and the subspace based power estimation steps, in the third section we elaborate an alternative approach using a two dimensional linear system where the matrix of the system is based on data retrieved from covariance matrix and beam forming spectrum. In the fourth section, we present a numerical evaluation and discuss the results.

\section{Signal Model}

We begin in this part be describing the signal model in the case of single radiating source, a uniform linear array of $N$ sensors in the far field region relatively to the source intercepts a planar wave front with azimuth angle $\theta$ measured with respect to the normal of the array (perpendicular line to the array elements), assuming that the emitted wave field and the sensors are vertically polarized and have the same operational frequency $f_{c}$, the signal vector at instant $t$ is written as:

$$
x(t)=s(t) a(\theta)+n(t)
$$

Where $\mathrm{t}=1, \ldots, \mathrm{K}$ and $s(t)$ is the source's envelope, $n(t) \in C^{N \times 1}$ is noise model which is complex random process, the steering vector $a(\theta) \in C^{N \times 1}$ contains the phase information, relatively the first element, it can be written by the following form:

$$
a(\theta)=\left(\begin{array}{c}
1 \\
e^{-j 2 \pi d \lambda^{-1} \sin \theta} \\
\vdots \\
e^{-j 2 \pi d(N-1) \lambda^{-1} \sin \theta}
\end{array}\right)
$$

Where $\lambda$ is the wavelength and $d$ is the consecutive distance between sensors. The second order statistics of data is given by the relation:

$$
\Gamma=<x x^{+}>=\sigma_{1}^{2} a(\theta) a^{+}(\theta)+\sigma^{2} I_{N}
$$

Where $<_{\text {. }}>$ denotes the time average, $\sigma_{1}^{2}$ is the source's power, $\sigma^{2}$ is the noise power and $I_{N}$ is the identity matrix. Most of high resolution angular spectra [8] rely on $\Gamma$ to estimate the parameters of the sources, the metric used to quantify the level of signal over noise powers is the Signal to Noise Ratio (SNR) given in decibel $(\mathrm{dB})$, it is defined by the following relation: 


$$
S N R=10 \log _{10}\left(\frac{\sigma_{1}^{2}}{\sigma^{2}}\right)
$$

Let us begin the analysis of the noise-free case $n(t)=0_{N \times 1}$, the spectral matrix is reduced into $\Gamma=\sigma_{1}^{2} a(\theta) a^{+}(\theta)$ which is rank one operator, it is obvious that spectrum in this case contains one non zero eigenvalue which is $\sigma_{1}^{2} a^{+} a=N \sigma_{1}^{2}$, therefore the spectrum is described by the vector:

$$
\sigma_{\Gamma}=\left[N \sigma_{1}^{2}, 0_{1 \times N-1}\right]
$$

Using the eigendecomposition, it is possible to compute the signal power, in the presence of perturbing noise, the spectrum of covariance matrix is given by the relation:

$$
\sigma_{\Gamma}=\left(\begin{array}{c}
\lambda_{1} \\
\lambda_{2} \\
\vdots \\
\lambda_{N}
\end{array}\right)=\left(\begin{array}{c}
N \sigma_{1}^{2}+\sigma^{2} \\
\sigma^{2} \\
\vdots \\
\sigma^{2}
\end{array}\right)
$$

After the eigendecomposition, an accurate estimation of source power is possible using the following equation:

$$
\sigma_{1}^{2}=\frac{\lambda_{1}-\frac{\sum_{j=2}^{N} \lambda_{j}}{N-1}}{N}=\frac{(N-1) \lambda_{1}-\sum_{j=2}^{N} \lambda_{j}}{N(N-1)}
$$

Where the noise power estimation is obtained from $N-1$ eigenvalues:

$$
\sigma^{2}=\frac{\sum_{j=2}^{N} \lambda_{j}}{N-1}
$$

The equations (7) and (8) are the subspace based solution of the power estimation, reduced to the case of single source. In the next section we propose a new formalism using angular spectrum obtained from beam forming function.

\section{New Solution of Power Estimation of One Source}

In this section, we explain a new method of power estimation of single source without using the spectral decomposition of the covariance matrix of received data, the solution is based on the angle of incidence $\theta$ of the wave front relatively to the normal of the array, a standard beam forming spectrum is sufficient in this case to obtain an estimate of $\theta$. Power estimation is based on two criteria, the value of the peak of beam forming spectrum and the trace of the covariance matrix. We begin with the expression of the trace, the signal model at the $i^{t h}$ sensor is:

$$
x_{i}(t)=e^{-j 2 \pi d(i-1) \lambda^{-1} \sin (\theta)} s(t)+n_{i}(t)
$$

Based on the hypothesis of spatially and temporally ergodic noise field $n(t)$, the diagonal elements of $\Gamma$ are:

$$
\Gamma_{i i}=<x_{i}(t) x_{i}^{*}(t)>=<s(t) s^{*}(t)>+<n_{i}(t) n_{i} *(t)>=\sigma_{1}^{2}+\sigma^{2}
$$

Given that the covariance matrix is evaluated using finite number of samples $K$, an estimate of the sum of the two powers is simply the mean eigenvalue given by the relation:

$$
<\lambda>=\frac{\operatorname{Tr}(\Gamma)}{N}=\frac{1}{N} \sum_{i}^{N} \Gamma_{i i}=\sigma_{1}^{2}+\sigma^{2}
$$


Next we explicit the theoretical expression of the beam forming 's peak value. For a given angle $\theta$ in the visible region of the array $\Omega=\left[\theta_{\min }, \theta_{\max }\right]$, the beam forming function is calculated using scan vector $b(\theta)$ written as:

$$
f(\theta)=\frac{1}{N^{2}} b^{+}(\theta) \Gamma b(\theta)=\frac{1}{N^{2}} b^{+}(\theta)\left(\sigma_{1}^{2} a(\theta) a^{+}(\theta)+\sigma^{2} I_{N}\right) b(\theta)
$$

If the test vector $b(\theta)$ coincides with the directional vector of the source $a(\theta)$, the function reaches its maximum value:

$$
\operatorname{Max}\{f(\theta)\}=\frac{1}{N^{2}} a^{+}(\theta)\left(\sigma_{1}^{2} a(\theta) a^{+}(\theta)+\sigma^{2} I_{N}\right) a(\theta)
$$

In this case, the value of the peak is simplified into the following relation:

$$
\operatorname{Max}\{f(\theta)\}=\frac{\sigma_{1}^{2}}{N^{2}} a^{+}(\theta) a(\theta) a^{+}(\theta) a(\theta)+\frac{\sigma^{2}}{N^{2}} a^{+}(\theta) I_{N} a(\theta)=\sigma_{1}^{2}+\frac{\sigma^{2}}{N}
$$

Let us consider a two dimensional vector of signal and noise powers defined by:

$$
X=\left(\begin{array}{l}
\sigma_{1}^{2} \\
\sigma^{2}
\end{array}\right)
$$

Combining the two metrics of trace and peak value, we can build a two dimensional linear system $Y=A X$ relating the two powers as the following:

$$
Y=A X=\left(\begin{array}{c}
\operatorname{Max}\{f(\theta)\} \\
\frac{\operatorname{Tr}(\Gamma)}{N}
\end{array}\right)=\left(\begin{array}{cc}
1 & \frac{1}{N} \\
1 & 1
\end{array}\right)\left(\begin{array}{l}
\sigma_{1}^{2} \\
\sigma^{2}
\end{array}\right)
$$

The solution of the above equation is obtained using the inverse of the matrix $A$ as the following:

$$
X=A^{-1} Y=\left(\begin{array}{cc}
\frac{-N}{1-N} & \frac{1}{1-N} \\
\frac{N}{1-N} & \frac{-N}{1-N}
\end{array}\right)\left(\begin{array}{c}
\operatorname{Maxf}(\theta) \\
\frac{\operatorname{Tr}(\Gamma)}{N}
\end{array}\right)
$$

Next, the proposed solution consists of the following steps:

- Estimate the covariance matrix $\Gamma$.

- Compute the mean eigenvalue $<\lambda>$ using the trace.

- Find the peak of the beam forming $\operatorname{Max}\{f(\theta)\}$.

- Solve the linear system given by equation (16).

Finally, the SNR is obtained by the components of the solution vector $X$ :

$$
S N R=10 \log _{10}\left(\frac{X_{1}}{X_{2}}\right)
$$

The main relation of the beam forming based power estimation is the equation (17), since we assumed the existence of single source, then the spatial function $f(\theta)$ is sufficient to scan the visible region and estimate the angle of incidence of the source, however in the presence of multiple sources as primary and interfering wave fronts, one must employ other techniques to estimates the power parameter.

For performance evaluation, we choose the numerical approach, in the next section, we present some results of the simulation comparatively to the subspace based method. 


\section{Numerical Evaluation}

In this part, we perform a numerical simulation of power estimation of single radiating source using an array of isotropic sensors that are equally spaced, the source waveform is modeled based on narrowband assumption (slowly varying envelope approximation). We consider a carrier frequency of $v=1500 \mathrm{MHz}$, given the wave speed $c \square 3 \times 10^{8} \mathrm{~m} / \mathrm{s}$, the corresponding wavelength is $\lambda=c / v=0.2 \mathrm{~m}$. The source waveform is given by linear frequency modulation where it starts with frequency $v_{1}=100 \mathrm{~Hz}$ and ends with frequency $v_{2}=250 \mathrm{~Hz}$ where the bandwidth is $\Delta v=150 \mathrm{~Hz}$ and the narrowband condition is verified:

$$
\Delta v \square v
$$

The expression of the waveform is generated for period of $\mathrm{T}=2 \mathrm{~s}$, it is given by the following expression:

$$
s(t)=\sin (2 \pi t(\alpha t+\beta)) e^{-j 2 \pi v t}
$$

Where the constants $\alpha$ and $\beta$ are fixed by:

$$
\left\{\begin{array}{c}
\alpha=\frac{v_{2}-v_{1}}{T} \\
\beta=v_{1}
\end{array}\right.
$$

We suppose that the collected echoes by the sensors are converted into baseband signals by removing the carrier expression $e^{-j 2 \pi v t}$, the sampling frequency is $F_{s}=1 \mathrm{kHz}$ which corresponds to $K=2000$ samples.

The array is composed of $N=4$ dipoles where the inter-element distance is half the operational wavelength $d=\lambda / 2=20 \mathrm{~cm}$. the sensors and the radiating sources are placed in the same horizontal plane where the angle of incidence using the far-field condition (planar wave front) is $\theta=15^{\circ}$ relatively to the normal of the array.

Figure 1 represents the frequency spectrum obtained from data of the first sensor using Fourier transform:

$$
F(v)=\int_{R} x_{1}(t) e^{-j 2 \pi v t} d t
$$

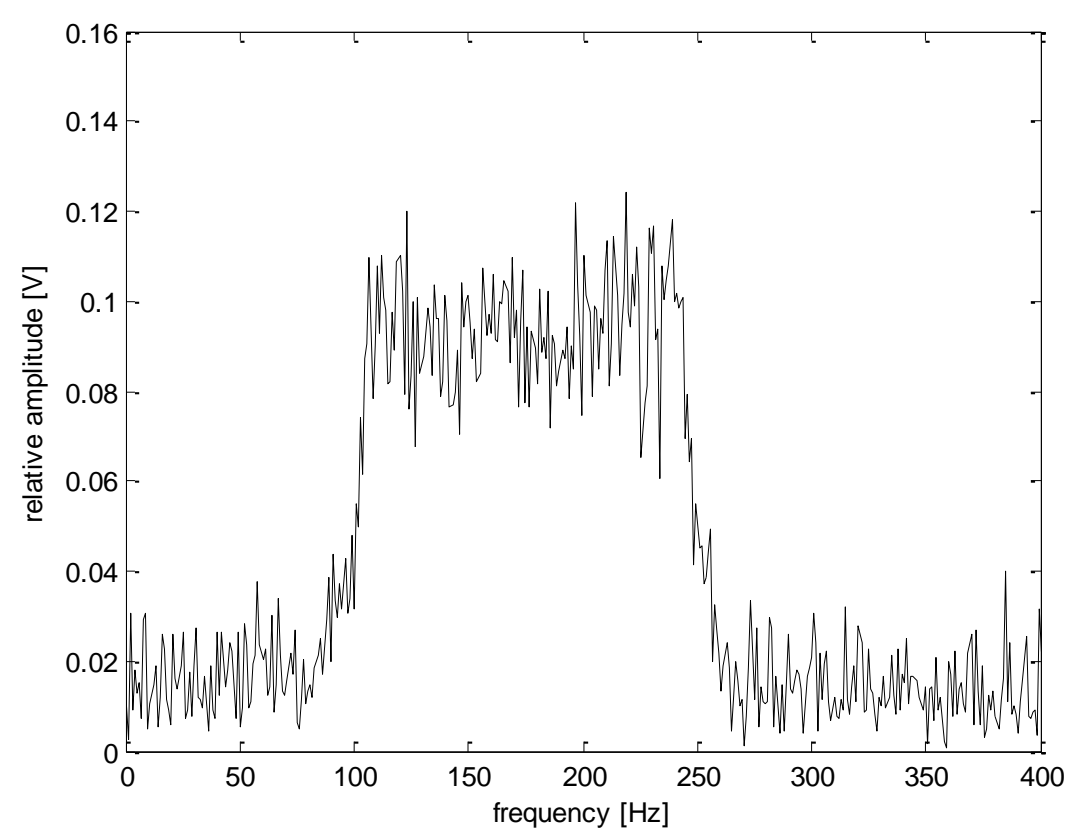

Figure 1. Frequency Spectrum of Baseband Waveform 
For the power estimation, we fix the noise power at $\sigma^{2}=0.25 \mathrm{~W}$ and we vary the source power starting from $\sigma_{1}^{2}=0.01$ to the value $2.5 \mathrm{~W}$ with step of approximately $0.1 \mathrm{~W}$, these values correspond to the range $S N R=-14 \mathrm{~dB}$ and $S N R=10$ $\mathrm{dB}$. For each value of signal strength, we generate the data $X(t)$ and estimate the $S N R$ over $L=100$ trials, we compare the performance of the subspace based method (Equation 7) and the alternative solution (Equation 17), the results are presented in Figure 2 .

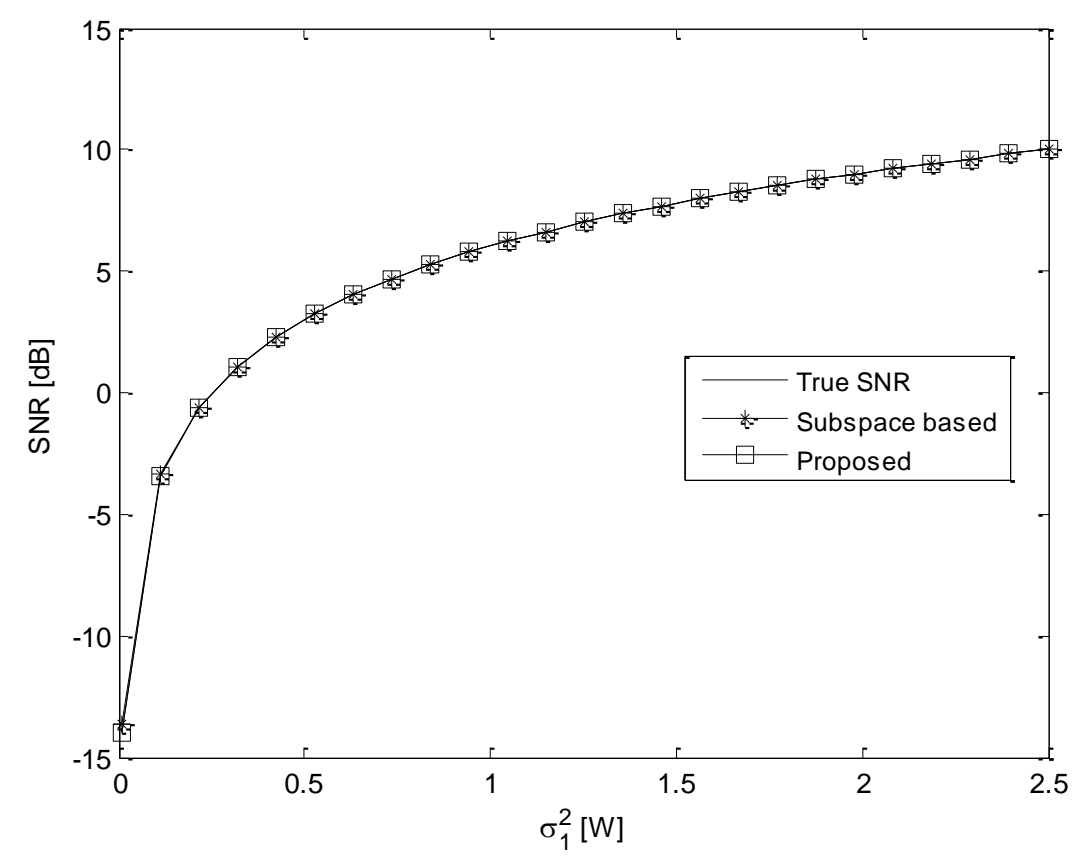

Figure 2. SNR Estimation of Single Radiating Source, $\sigma^{2}=0.25 \mathbf{W}$

We remark that the two solutions have identical performances where the parameters of the wave front are $\left(\sigma_{1}^{2}, \theta\right)$, the performance of the beam forming based solution is the same for any direction of the wave front $\theta \in \Omega$ where $\Omega=[-\pi / 2,+\pi / 2]$ in the case of uniform linear array, indeed, single peak detection is an easy procedure for single emitter.

\section{Conclusion}

Among the applications of signal array processing is the power estimation of radiating sources based on received data, in the case of multiple sources, data processing needs to mitigate the interferences between individual waveforms. In this paper, we have discussed a particular case of single radiating source where the parameter of interest if the signal over noise level which is also called signal to noise ratio. We have presented an alternative solution of power estimation using the standard beam forming and the trace of spatial covariance matrix of collected data, the proposed solution is based on far-field condition and narrowband spectrum. For performance analysis, we have conducted some computer simulations where we compared two solutions of SNR estimation for different values of signal strength. The obtained results for sufficient number of trials demonstrated that the eigenvalue based and the proposed methods are identical in terms of estimation accuracy. 


\section{Appendix}

In this part, we present the power estimation method based on subspace decomposition in the case of multiple sources. Let the number of source to be $P$ with the condition $N>P$. Firstly, we suppose that noise field is spatially and temporally uncorrelated, secondly, we assume that the waveforms of the sources are uncorrelated but may have different powers, the correlation matrix $\Gamma_{s s}$ is diagonal as the following:

$$
\Gamma_{s s}=\left(\begin{array}{cccc}
<s_{1}(t) s_{1}^{*}(t)> & 0 & \ldots & 0 \\
0 & <s_{2}(t) s_{2}^{*}(t)> & \ldots & 0 \\
\ldots & \ldots & \ldots & \ldots \\
\ldots & \ldots & \ldots & <s_{P}(t) s_{P}^{*}(t)>
\end{array}\right)
$$

Where $\sigma_{i}^{2}=<s_{i}(t) s_{i}^{*}(t)>$ and the covariance matrix is $\Gamma=A \Gamma_{s s} A^{+}+\sigma^{2} I_{N}$. The steering matrix has the dimensions $A \in C^{N \times P}$. Using high resolution spectral technique to estimate the parameters $\left\{\theta_{1}, \theta_{2}, \ldots, \theta_{P}, \sigma^{2}\right\}$ and reconstruct the steering matrix $A$, the source correlation matrix is estimated by the following steps [9]:

$$
\begin{aligned}
& A \Gamma_{s s} A^{+}=\Gamma-\sigma^{2} I_{N} \\
& \Gamma_{s s}=\left(A^{+} A\right)^{-1} A^{+}\left(\Gamma-\sigma^{2} I_{N}\right) A\left(A^{+} A\right)^{-1} \\
& \Gamma_{s s}=A^{?}\left(\Gamma-\sigma I_{N}\right)\left(A^{?}\right)^{+}
\end{aligned}
$$

\section{References}

[1] R. Shevgaonkar, "Electromagnetic Waves", McGraw Hill Education, (2005).

[2] F. Gross, "Smart Antennas for Wireless Communications", McGraw-Hill, Professional, (2005).

[3] A. De, T. K. Sarkar and M. Salazar-Palma, "Characterization of the Far-Field Environment of Antennas Located Over a Ground Plane and Implications for Cellular Communication Systems", in IEEE Antennas and Propagation Magazine, vol. 52, no. 6, (2010), pp. 19-40.

[4] D. Rodrigo, B. A. Cetiner and L. Jofre, "Frequency, Radiation Pattern and Polarization Reconfigurable Antenna Using a Parasitic Pixel Layer", in IEEE Transactions on Antennas and Propagation, vol. 62, no. 6, (2014), pp. 3422-3427.

[5] N. Wang, P. Agathoklis and A. Antoniou, "A new DOA estimation technique based on subarray beamforming", in IEEE Transactions on Signal Processing, vol. 54, no. 9, (2006), pp. 3279-3290.

[6] M. Sahmoudi and M. G. Amin, "Optimal Robust Beamforming for Interference and Multipath Mitigation in GNSS Arrays", 2007 IEEE International Conference on Acoustics, Speech and Signal Processing - ICASSP '07, Honolulu, HI, (2007), pp. III-693-III-696.

[7] J. Wang, Y. Zhao and Z. Wang, "A MUSIC like DOA estimation method for signals with low SNR", 2008 Global Symposium on Millimeter Waves, Nanjing, (2008), pp. 321-324.

[8] Y. Khmou, S. Safi and M. Frikel, "Comparative Study between Several Direction Of Arrival Estimation Methods", Journal of Telecommunications and Information Technology, (2014), pp.41-p.48.

[9] J. Lardiès, H. Ma and M. Berthillier, "Power Estimation of Acoustic Sources by Sensor Array Processing", Open Journal of Acoustics, vol. 3, no. 2A, (2013), pp. 1-7. 
International Journal of Hybrid Information Technology

Vol. 10, No.11 (2017) 